\title{
Comunicação
}

\section{Ocorrência de Compsosoma perpulchrum em eucalipto, no Estado de Minas Gerais, Brasil}

\author{
Gláucia Cordeiro ${ }^{1}$, Norivaldo dos Anjos ${ }^{2}$, Iris Cristiane Magistrali ${ }^{3}$ e Pedro Guilherme Lemes ${ }^{4}$
}

\section{RESUMO}

As espécies do gênero Eucalyptus são as mais utilizadas no setor florestal graças, principalmente, ao seu rápido crescimento e à sua boa adaptação às diferentes condições de solo e de clima, no Brasil. Entretanto, alguns insetos podem-se tornar pragas dessa espécie florestal, como é o caso de Compsosoma perpulchrum (Coleoptera: Cerambycidae), que apresenta registro roletando árvores de E. grandis no Paraná. Este trabalho teve como objetivo caracterizar o dano de C. perpulchrum em plantio comercial de eucalipto, no município de Paula Cândido-MG. Em março de 2008, foi vistoriado o plantio, cujas árvores e galhos roletados foram mensurados. As árvores danificadas eram de E. urophylla e a maioria dos roletamentos ocorreram no terço mediano. O comprimento e o diâmetro dos galhos roletados foram de $0,94 \pm 0,04 \mathrm{~m}$ e de $0,90 \pm 0,06 \mathrm{~cm}$, respectivamente. O comprimento da faixa de roletamento foi $3,63 \pm 0,18 \mathrm{~cm}$. C. perpulchrum roleta a parte média das árvores de E. urophylla, causando perda da sua dominância apical. Este é o primeiro relato da ocorrência desse serrador, danificando plantio comercial de E. urophylla no Estado de Minas Gerais.

Palavras-chave: Praga florestal, injúria, cerambycidae, serrador.

\section{ABSTRACT}

\section{Occurrence of Compsosoma perpulchrum in an eucalypt stand, in Minas Gerais State, Brazil}

The species of genus Eucalyptus are the most used in the forestry sector, mostly because of its fast growth and adaptation to the different conditions of soil and climate in Brazil. However, a few insects, such as Compsosoma perpulchrum (Vigors, 1825) (Coleoptera: Cerambycidae) can become pests of girdled E. grandis Hill ex. Maiden in the State of Paraná. The objective of this work was to report the occurrence of $C$. perpulchrum in an eucalypt stand in the municipality of Paula Cândido, Minas Gerais State, Brazil. Girdled trees and branches were inspected and measured in March, 2008. The damaged trees were E. urophylla S. T. Blake and most of the girdle occurred in the middle third of the tree height. The length of the girdle range was $3.63 \pm 0.18 \mathrm{~cm}$. The length and diameter of the girded branches were $0.94 \pm 0.04 \mathrm{~m}$ and $0.90 \pm 0.06 \mathrm{~cm}$, respectively. This is the first report of the insect damage to this eucalypt species in the State of Minas Gerais.

Key words: Forest pest, insect injury, cerambycidae, twig girdler.

\footnotetext{
Recebido para publicação em 21/06/2010 e aprovado em 20/07/2011

Engenheira Florestal, Doutoranda em Entomologia/UFV. Departamento de Biologia Animal/Entomologia/Casa dos Cupins. Universidade Federal de Viçosa. Av. P.H. Rolfs S/N. Viçosa-MG. 36570-000. glaucordeiro@gmail.com (Autor para correspondência) Bolsista CNPq

${ }^{2}$ Engenheiro Florestal, Pós-Doutor, Departamento Entomologia/Casa dos Cupins. Universidade Federal de Viçosa. Av. P.H. Rolfs S/N. Viçosa-MG. 36570-000. nanjos@ufv.br ${ }^{3}$ Engenheiro Florestal, Mestrando em Entomologia/UFV. Departamento Entomologia/Casa dos Cupins. Universidade Federal de Viçosa. Av. P.H. Rolfs S/N. Viçosa-MG. 36570000. pedrolemes@hotmail.com

${ }^{4}$ Engenheira Florestal, Departamento Entomologia/Casa dos Cupins. Universidade Federal de Viçosa. Av. P.H. Rolfs S/N. Viçosa-MG. 36570-000. irismagistrali@gmail.com
} 


\section{INTRODUÇÃO}

As culturas florestais homogêneas têm sido plantadas para minimizar o desmatamento e propiciar fontes de madeira para diversos fins. As espécies do gênero Eucalyptus são as mais utilizadas, graças, principalmente, ao seu rápido crescimento e à sua boa adaptação às diferentes condições de solo e de clima, no Brasil. Em 2010, o país contava com, aproximadamente, 4,7 milhões de hectares de eucaliptais, sendo $29,4 \%$ desta área no Estado de Minas Gerais (Associação Brasileira de Produtores de Florestas Plantadas, 2011). A eucaliptocultura brasileira é responsável pelo abastecimento de matéria prima para as indústrias de papel, celulose e siderurgia, entretanto, essa cultura enfrenta graves problemas com relação a pragas (Santos et al., 2000).

No Estado de Minas Gerais, existiam 34 espécies de insetos daninhos, distribuídas nas ordens Lepidoptera, Coleoptera e Orthoptera, sendo estes pertencentes a 22 famílias e associados ao gênero Eucalyptus (Berti Filho, 1981). Os insetos associados ao eucalipto, para o Brasil, estavam distribuídos em oito ordens, com 60 famílias e aproximadamente 200 espécies (Berti Filho, 1997). A ordem Coleoptera era representada por 73 espécies, sendo 49,32\% pertencentes à família Cerambycidae, na qual estão incluídos os serradores do gênero Compsosoma Lepeletier \& Audinet-Servile, 1830. Além das espécies deste gênero, existem outros serradores pertencentes aos gêneros Oncideres Lepeletier \& Audinet-Servile, 1830, e Psyllotoxus Thomson, 1868, que danificam os eucaliptos no Brasil, conforme afirmaram Monné (2002) e Mariconi (1969).

Os serradores, ao roletarem ramos em pleno vigor, podem causar a morte ou modificar o crescimento das árvores, originando forquilhas típicas, denunciadoras da ação da praga (Baucke, 1959).

Em vista da importância dessa praga, causadora de prejuízos relevantes para a eucaliptocultura no Brasil, este trabalho teve como objetivo registrar a ocorrência de $C$. perpulchrum (Vigors, 1825 - Figura 1) em um plantio de eucalipto, em Paula Cândido-MG

\section{MATERIAL E MÉTODOS}

A caracterização do dano de C. perpulchrum (Figura 2) foi realizada com base em vistoria em plantio de Eucalyptus urophylla S. T. Blake, localizado no lugarejo denominado Santa Rosa, zona rural do município de Paula Cândido - MG, no início do mês de março de 2008. O plantio possuía três meses de idade, espaçamento $3 \times 2 \mathrm{me}$ área total de 12 hectares. A vizinhança do plantio era constituída de pastagens e, para além destas, fragmentos de Mata Atlântica.

Todo o plantio foi vistoriado, visando a delimitar o foco de ocorrência dos danos. Neste foco, foram anota- das as quantidades de árvores danificadas e não danificadas. Entre as danificadas, foram mensuradas dez árvores, aleatoriamente, quanto ao diâmetro do coleto, altura total e altura do roletamento, usando-se fita milimétrica. Foi anotada, também, a posição em relação à altura do roletamento na árvore (terço inferior, mediano ou superior). Dez galhos injuriados foram coletados e mensurados quanto ao comprimento total, diâmetro e quanto ao comprimento da faixa de roletamento, utilizando-se fita milimétrica. Foi observado, nestes galhos, se havia incisões de posturas e sinais de alimentação.

\section{RESULTADO E DISCUSSÃO}

A espécie de eucalipto danificado pelo serrador $C$. perpulchrum foi diferente da registrada por Souza et al. (1995), que era Eucalyptus grandis Hill ex Maiden.

O foco das árvores danificadas pelo serrador foi constatado nas margens do plantio, próximo à pastagem; das 150 árvores observadas, 40 estavam danificadas. Das árvores roletadas, $72,5 \%$ estavam localizadas da terceira à quinta linha de plantio e $26,67 \%$ apresentaram o ponteiro principal roletado (Figura 2). A maioria (70\%) dos roletamentos foram realizados no terço mediano das árvores e não foi observado roletamento no terço superior.

\section{$1,8 \mathrm{~cm}$}
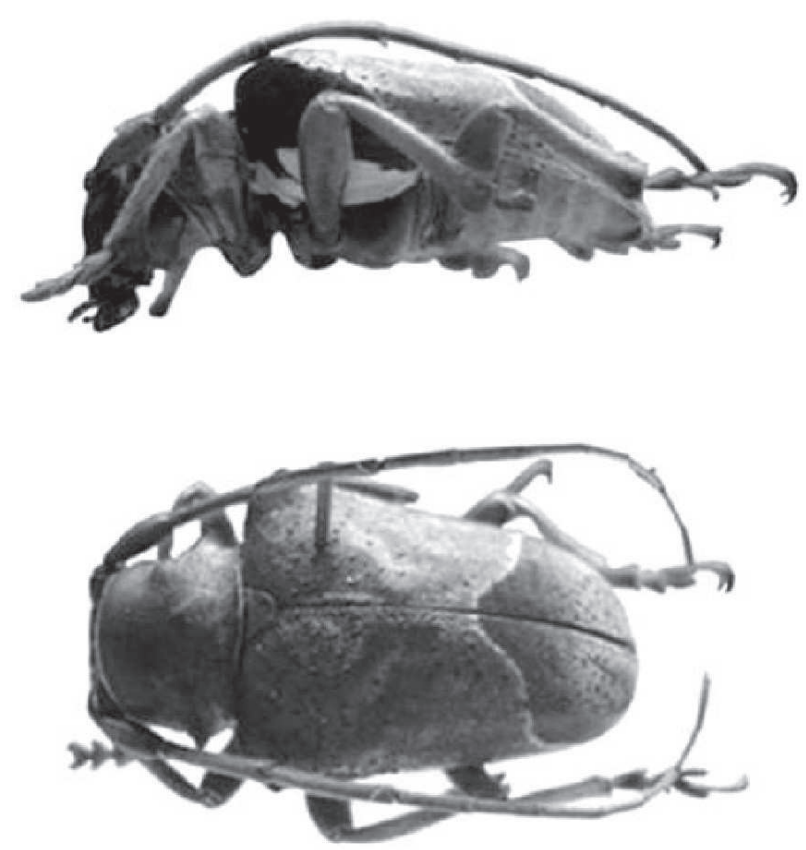

Cordeiro, 2008

Figura 1. Adulto de Compsosoma perpulchrum (Vigors, 1825). Paula Cândido - MG. 2008. Arte: Lemes, 2008. 

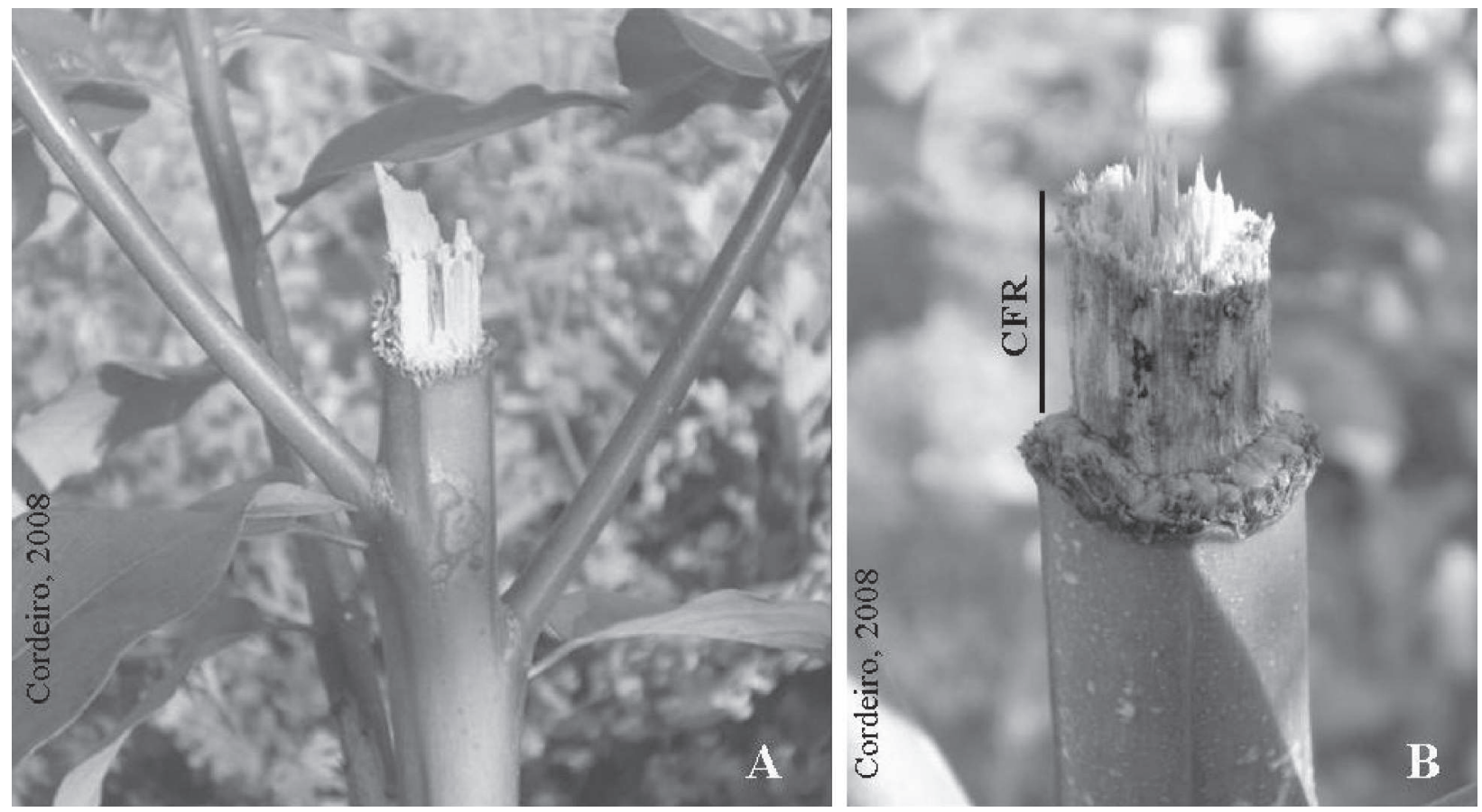

Figura 2. Eucalyptus urophylla S.T. Blake, roletado por Compsosoma perpulchrum (Vigors, 1825). Em B, detalhe do comprimento da faixa de roletamento (CRF). Paula Cândido - MG. 2008. Arte: Lemos, 2008.

A extensão do foco registrado no Estado de Minas Gerais foi inferior àquela registrada por Souza et al. (1995) no Paraná, que abrangeu cerca de 900 árvores com um ano de idade.

O diâmetro, à altura do coleto das árvores danificadas, foi de $2,48 \pm 0,17 \mathrm{~cm}$ e as alturas, das árvores e dos galhos roletados, foram de $1,25 \pm 0,07 \mathrm{~m} \mathrm{e} 0,53 \pm 0,07 \mathrm{~cm}$; respectivamente. Esta altura de roletamento é inferior à relatada para serradores do gênero Oncideres, em árvores da família Fabaceae (Oliveira, 2007; Cordeiro, 2008), uma vez que ainda não existem informações para $C$. perpulchrum.

O comprimento total e o diâmetro dos galhos roletados foram de $0,94 \pm 0,04 \mathrm{~m}$, variando de 0,75 a $1,12 \mathrm{~m}$, e de $0,90 \pm 0,06 \mathrm{~cm}$, variando de 0,57 a $1,27 \mathrm{~cm}$, respectivamente. Estes valores foram inferiores ao relatado por Coutinho et al. (1998), Magistrali et al. (2008) e Cordeiro (2008) para $O$. saga, em hospedeiros pertencentes à família Fabaceae. De acordo com Souza et al. (1995), adultos de $C$. perpulchrum roletaram galhos de $E$. grandis com, aproximadamente, $2,0 \mathrm{~cm}$ de diâmetro, ou seja, diâmetro superior ao observado neste trabalho. $\mathrm{O}$ comprimento da faixa de roletamento (Figura 2B) foi de $3,63 \pm 0,18 \mathrm{~cm}$, correspondendo a $3,86 \%$ do comprimento total do galho roletado.

Segundo Giacomel (1979), que, entretanto, não menciona o hospedeiro, a fêmea de $C$. perpulchrum escolhe um ramo para a postura, remove a casca formando um círculo em torno deste e insere o ovo, No presente trabalho, em E. urophylla, todavia, não foram encontrados sinais de posturas.
De acordo com Baucke (1959) e Amante et al. (1976), a casca dos galhos tenros é a principal fonte de alimentação dos besouros serradores, mas, neste caso, somente dois ponteiros roletados apresentaram cascas roídas.

\section{CONCLUSÃO}

Conclui-se que $C$. perpulchrum roleta a parte média das árvores de E. urophylla, causando perda da sua dominância apical e esta é a primeira vez que se relata a ocorrência deste serrador, danificando plantio comercial de E. urophylla, em Minas Gerais.

\section{AGRADECIMENTOS}

Os autores agradecem ao CNPq pelo apoio financeiro.

\section{REFERENCIAS}

Amante E, Berlato MA \& Gessinger GI (1976) Biologia do "Serrador" da acácia-negra, Oncideres impluviata (Germar, 1824) (Coleoptera: Cerambycidae) no Rio Grande do Sul, I Etologia. Agronomia Sulriograndense, 12:3- 56.

Associação Brasileira de Produtores de Florestas Plantadas (2011) Anuário estatístico da ABRAF: ano base 2010. Brasília, 130p. Disponível em: <http://www.abraflor.org.br/estatisticas/ ABRAF11/ABRAF11-BR.pdf>. Acesso em: 02 de junho de 2011.

Baucke O (1959) Notas sobre a biologia e o controle ao "serrador" da acácia negra. Agronomia Sulriograndense, 4:103-104.

Berti Filho E (1981) Insetos associados a plantações de espécies do gênero Eucalyptus nos estados da Bahia, Espírito Santo, Mato Grosso do Sul, Minas Gerais e São Paulo. Tese de Doutorado. Escola Superior de Agricultura "Luiz de Queiroz", Piracicaba, 175p. 
Berti Filho E (1997) Impacto de Coleoptera Cerambycidae em florestas de Eucalyptus no Brasil. Scientia Forestalis, 52:51-54.

Coutinho CL, Carvalho AC, Oliveira ES \& Veiga BGA (1998) Oncideres saga (Dalman, 1823) (Coleoptera: Cerambycidae) e a arborização urbana em Seropédica, RJ. Floresta e Ambiente, 5:50-54.

Giacomel F (1979) Sobre a biologia de Compsosoma perpulchrum e Compsosoma phaleratum (Coleoptera, Cerambycidae). Dusenia, 11:95-101.

Cordeiro G (2008) Aspectos biológicos de Oncideres saga (Dlaman) (Coleoptera: Cerambycidae) e efeitos de seus danos em Acacia mangium Willd. Dissertação de Mestrado. Universidade Federal de Viçosa, Viçosa, 82p.

Magistrali IC, Magistrali PR, De Nadai J \& Goellner AA (2008) Parâmetros biológicos de Oncideres saga (Dalman, 1823) (Coleoptera: Cerambycidae) em Acacia mearnsii De Willd. Revista Trópica, 2:3-10.
Mariconi RC (1969) Sobre alguns Cerambycidae (Coleoptera) e suas plantas hospedeiras. Ciência e Cultura, 21:470.

Monné MA (2002) Catalogue on the Neotropical Cerambycidae (Coleoptera) with known host plant. Part IV: Subfamily Lamiinae, tribes Batocerini to Xenofreini. Publicações Avulsas do Museu Nacional, 94:1-92.

Oliveira LS (2007) Aspectos entomológicos em povoamentos homogêneos de Acacia mearnsii De Willd. Tese de Doutorado. Universidade Federal de Santa Maria, Santa Maria, 121p.

Santos GP, Zanuncio TV \& Zanuncio JC (2000) Desenvolvimento de Thyrinteina arnobia Stoll (Lepidoptera: Geometridae) em folhas de Eucalyptus urophylla e Psidium guajava. Anais da Sociedade Entomológica do Brasil, 29:13-22.

Souza NJ, Correa RM, Pedrosa-Macedo JH, Marques EM \& Bittencourt SJA (1995) Anelamento de árvores de Eucalyptus grandis, causado por Compsosoma perpulchrum (Vigors, 1825) (Coleoptera: Cerambycidae). Agrárias, 14:231-233. 